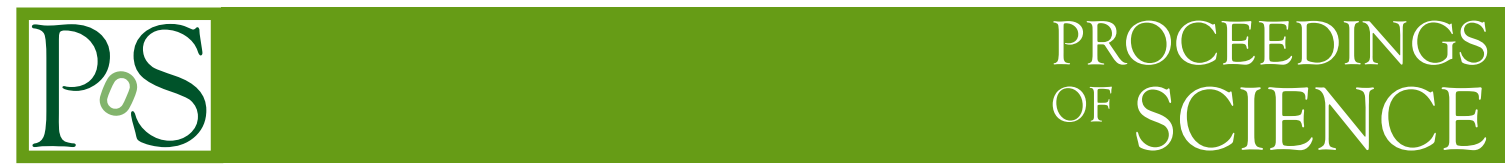

\title{
Polarized Solid Targets at Jefferson Lab
}

\section{C.D. Keith*}

Thomas Jefferson National Accelerator Facility, Newport News VA, USA

E-mail: ckeithejlab.org

For nearly two decades, polarized solid targets have played a vital role in the nuclear physics program at Jefferson Lab and were successfully utilized in each of the lab's three experimental halls during its $6 \mathrm{GeV}$ era. Following a review of their $6 \mathrm{GeV}$ operation, I briefly discuss the role that polarized solid targets will play in JLab's future at $12 \mathrm{GeV}$.

XVIth International Workshop in Polarized Sources, Targets, and Polarimetry, PSTP2015,

14-18 September 2015,

Bochum, Germany

${ }^{*}$ Speaker. 


\section{Introduction}

The Thomas Jefferson National Accelerator Facility (Jefferson Lab, or JLab) is a US national laboratory located in Newport News, VA with a mission to perform basic research in mediumenergy nuclear physics using CEBAF, the Continuous Electron Beam Accelerator Facility. CEBAF was originally conceived with a maximum electron beam energy of $6 \mathrm{GeV}$, and could simultaneously provide beams to three experimental end stations: Halls A, B, and C. The physics program began in 1995 and paused in 2012 for a three-year-long, \$350M upgrade to double the beam energy to $12 \mathrm{GeV}$, upgrade the instrumentation of the three existing experimental halls, and add a fourth end station, Hall D.

During JLab's $6 \mathrm{GeV}$ era, polarized solid targets were used on twelve occasions, beginning in 1998 (in Hall C), and ending in 2012 with experiments in Hall A and Hall B. About one in seven experiments at JLab used a solid polarized target, and approximately $50 \mathrm{PhD}$ degrees were, (or will be) granted to students working on these experiments. Four such targets were used at $6 \mathrm{GeV}$, referred herein as the UVa target, the Hall B target, FROST, and the HDice target. Each is briefly described below.

\section{The UVa polarized target}

This target is so named because it was constructed in a collaborative effort between the University of Virginia (UVa), Jefferson Lab, and the University of Basel. It was used at the Stanford Linear Accelerator Center (SLAC) on three separate occasions and at JLab on four. The target is shown schematically in Figure 1 and is described in full detail by Averett et al.[1]. The key components for the target include a $5 \mathrm{~T}$ split-coil superconducting magnet, a high-cooling power ${ }^{4} \mathrm{He}$ evaporation refrigerator, and a multi-cell sample insert, all of which are optimized to operate the target with very high luminosities. CEBAF beam currents up to $140 \mathrm{nA}$ were used on $3 \mathrm{~cm}$ long targets of irradiated $\mathrm{NH}_{3}$ and $\mathrm{ND}_{3}$ to achieve luminosities of about $10^{36} \mathrm{~cm}^{-2} \mathrm{~s}^{-1}$. The entire system can be rotated $90^{\circ}$ to provide either longitudinal or transverse polarization.

This became the first polarized target, solid or gas, in operation at JLab, when it was used in 1998 to measure the electric form factor of the neutron $G_{n}^{e}$ [2]. Measurements of $G_{n}^{e}$ were continued during a second run period in 2001, and in 2002 the longitudinal and transverse spin structure functions of the proton and deuteron in the resonance region were measured [3]. In 2009 the target was again installed in Hall $\mathrm{C}$ for SANE, the Spin Asymmetries of the Nucleon Experiment, where the proton spin structure functions $g_{1}$ and $g_{2}$ were measured in the deep inelastic scattering regime [4]. Finally, the target was used in 2012 for the final $6 \mathrm{GeV}$ Hall A experiments. Here two experiments were performed concurrently: a measurement of the proton electric form factor $G_{p}^{e}$, and a determination of the proton spin structure function ratio $G_{E} / G_{M}[5,6]$. A considerable degree of maintenance and modification of the target was performed prior to the Hall A experiments, as described by Pierce et al. [7].

\footnotetext{
${ }^{1}$ This is the total luminosity for scattering from all nucleons in the target material.
} 


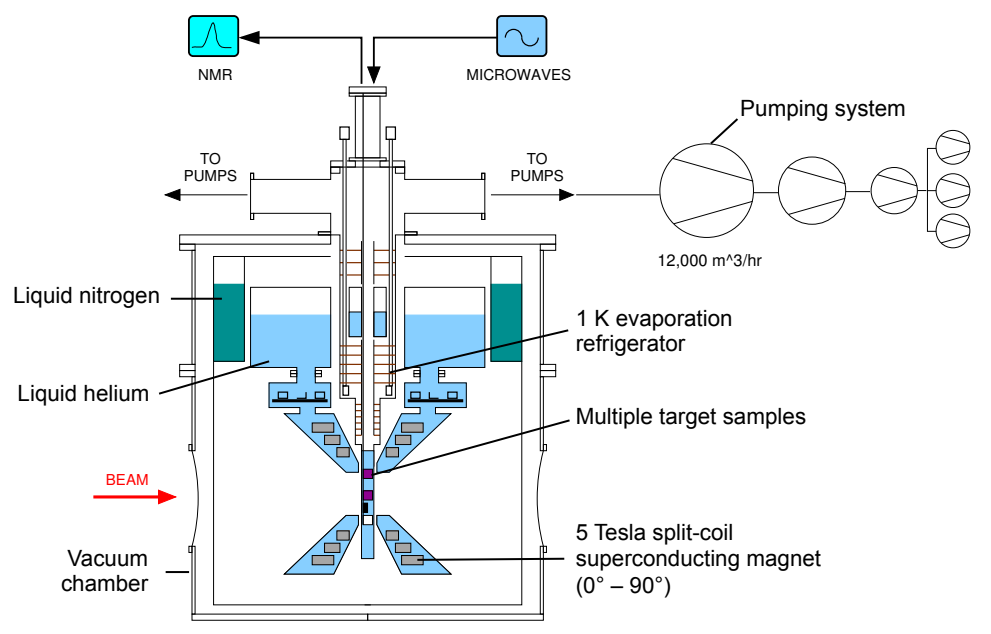

Figure 1: The UVa polarized solid target.

\section{The Hall B polarized target}

In the $6 \mathrm{GeV}$ era, the centerpiece of the Hall B instrumentation package was the CEBAF Large Acceptance Spectrometer (CLAS), a multi-gap, high-acceptance magnetic spectrometer in which the field was generated by six superconducting coils in a toroidal configuration [8]. This design left a field-free region at the center of the spectrometer which was suitable for the insertion of a dynamically polarized target. Such a target was utilized on multiple occasions between 1998 and 2009 to measure spin structure functions of the proton and deuteron [10,11], and target, beam, and double spin asymmetries in deeply virtual Compton scattering [12, 13, 14]. Electron beam currents on the $1 \mathrm{~cm}$ long ammonia samples were typically $2-10 \mathrm{nA}$, limited by the maximum permissible counting rate of the CLAS detector systems.

Like the UVa target, the Hall B polarized target was optimized for operation in nA electron beams and featured a $5 \mathrm{~T}$ superconducting split-coil magnet and a powerful ${ }^{4} \mathrm{He}$ evaporation refrigerator to polarize samples of irradiated $\mathrm{NH}_{3}$ and $\mathrm{ND}_{3}$. However, its geometrical design was significantly different in order to fit into the spectrometer, see Figure 2. As before, the ammonia samples were suspended vertically into a $1 \mathrm{~K}$ bath of liquid helium between the superconducting coils, using a motion-controlled sample insert. However, the liquid helium service dewar for the magnet was relocated two meters upstream from the superconducting magnet, and liquid for the $1 \mathrm{~K}$ bath was supplied from an Roubeau-style evaporation refrigerator oriented at an angle $25^{\circ}$ from the horizontal. Unlike the UVa target, the Hall B polarized target could only be used for longitudinal polarization.

\section{The frozen spin polarized target, FROST}

The previously described targets were continuously polarized at $1 \mathrm{~K}$ and $5 \mathrm{~T}$, a design that has been demonstrated to give good performance with charged-particle beams up to $10^{12}$ particles s $^{-1}$. Secondary beam experiments (e.g. tagged photons) must compensate for the lower beam intensity by maximizing the acceptance of scattered particles. Frozen spin targets are often chosen for this 


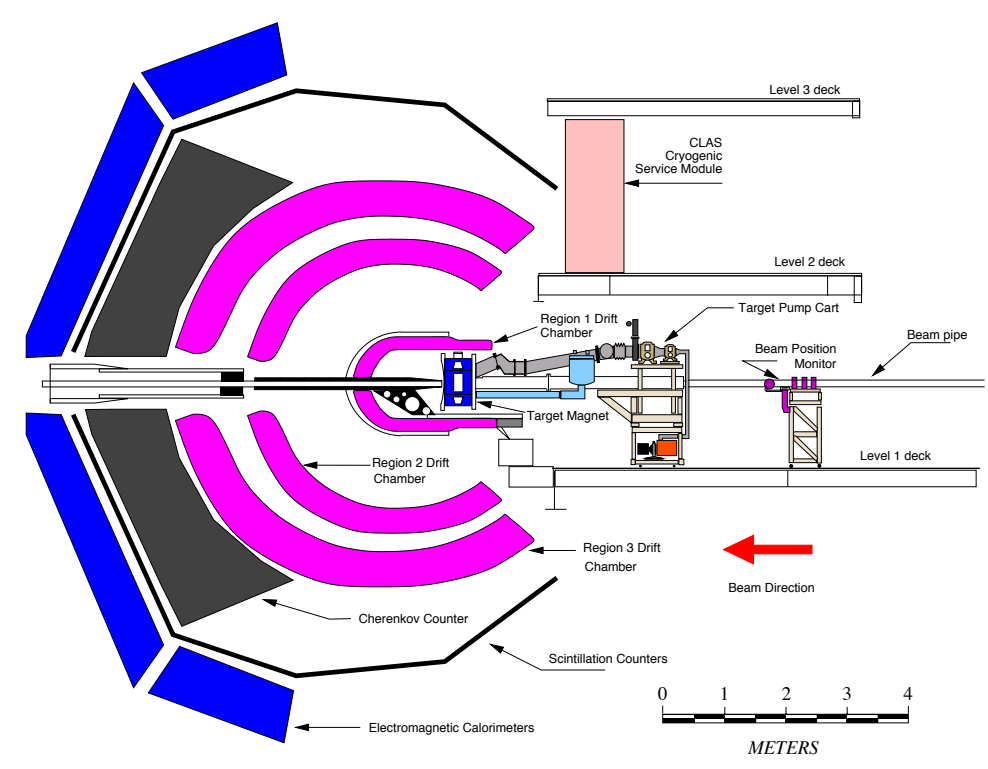

Figure 2: The Hall B polarized solid target.

role because they can operate in the beam without the large superconducting magnet necessary for dynamic polarization. The operation of a dynamically polarized, frozen spin target is depicted schematically in Figure 3. The target sample is dynamically polarized on a periodic basis using a high-field, high-uniformity magnetic field, while the scattering data is acquired as the polarization decays in a region of lower field.

A frozen spin, polarized proton target was constructed for use inside CLAS with beams of tagged photons [15]. The target sample, comprising frozen beads of butanol doped with the nitroxl radical TEMPO, was polarized outside CLAS at $250 \mathrm{mK}$ using a $5 \mathrm{~T}$ warm bore solenoid. Once polarized, the target cryostat was retracted from the solenoid and moved to the center of the spectrometer. Thin superconducting coils were installed inside the cryostat to maintain the target polarization during the experiment, a technique pioneered by the Bonn and Bochum target groups [17]. Polarizations up to $94 \%$ were obtained, and 1/e holding times of 2000-4000 hours were observed at temperatures down to about $25 \mathrm{mK}$.

The tagged photon experiments were grouped into two distinct run periods. In the first, the target polarization was maintained in the longitudinal orientation using a $0.56 \mathrm{~T}$ solenoidal holding field. A $0.50 \mathrm{~T}$ saddle coil was used to hold the target polarization transverse to the beam during the

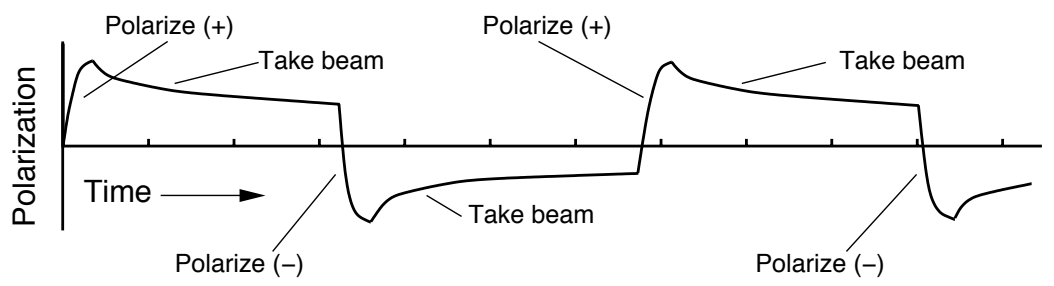

Figure 3: Operational sequence for a dynamically polarized, frozen spin target. 
second run period. In both instances, both circular and linear photon polarizations were utilized, resulting in a so-called "complete experiment" [16]. A record deuteron polarization of $-87 \%$ was also demonstrated with this frozen spin target, using fully deuterated propanediol doped with the trityl radical OX063.

\section{The HDice polarized target}

The use of solid deuterium hydride (HD) as a polarized target was pioneered in its current form by Honig, beginning in the 1967 [18], and further refined and realized by Sandorfi et al., first at Brookhaven National Laboratory and later at Jefferson Lab [19, 20]. Samples of solid HD are polarized under thermal equilibrium conditions of $15 \mathrm{~T}$ and about $15 \mathrm{mK}$. Small concentrations of molecular hydrogen and deuterium are added to the sample to increase the HD polarization rates via coupling with the $L=1$ rotational states of the $\mathrm{H}_{2}$ and $\mathrm{D}_{2}$ molecules. The sample is held at the low-temperature, high-field conditions for a few months, while the $L=1$ states of $\mathrm{H}_{2}$ and $\mathrm{D}_{2}$ decay to their $L=0$ ground states. Once the $L=1$ concentrations are sufficiently low, the HD relaxation rates become extraordinarily long, and the sample can be extracted from the high-field, low-temperature polarizing cryostat and used under more moderate conditions for low-intensity beam experiments.

Polarized deuterons in solid HD were utilized with tagged photons in Hall B to compliment the polarized proton experiments described in Section 4. The deuteron polarization (average value $20 \%$ ) was maintained in the longitudinal orientation by a $0.3 \mathrm{~T}$ solenoid installed inside the inbeam cryostat. Interweaved with the tagged photon experiment, a pair of tests using nA electron beams were performed to investigate the future viability of solid HD with such beams. The results are described by Lowry et al. [21].

\section{Future experiments with solid polarized targets}

At the time of this writing, there are more than one dozen approved or conditionally approved experiments that will utilize solid polarized targets at Jefferson Lab during the $12 \mathrm{GeV}$ era.

One experiment in Hall A will use the new SoLID ${ }^{2}$ spectrometer to examine transverse spin asymmetries in semi-inclusive deep inelastic scattering from polarized protons in the UVa target [22].

A new dynamically polarized target is under construction for the new large acceptance spectrometer in Hall B, CLAS12 [23]. The target is a collaborative effort between the Jefferson Lab Target Group, the University of Virginia, Old Dominion University, and Christopher Newport Universities. There are a total of six experiments approved for use with the target, primarily for protons and deuterons in $\mathrm{NH}_{3}$ and $\mathrm{ND}_{3}$, although polarized ${ }^{6} \mathrm{LiH}$ and ${ }^{7} \mathrm{LiD}$ samples will be used to examine the EMC effect in spin structure functions [24]. The target will be longitudinally polarized and is designed for luminosities up to $10^{35} \mathrm{~cm}^{-2} \mathrm{~s}^{-1}$.

Also in Hall B, there are three conditionally approved experiments to measure transverse spin asymmetries in semi-inclusive deep inelastic scattering and deeply virtual Compton scattering. The HDice target will be utilized for these experiments [25].

\footnotetext{
${ }^{2}$ Solenoidal Large Intensity Device
} 
An approved experiment Hall C will measure the initial state helicity correlation asymmetry $A_{L L}$ in Real Compton Scattering [26] from longitudinally polarized protons, and two conditionally approved experiments will make use of tensor polarized deuterons to measure the tensor structure function $b_{1}$ [27] and the tensor asymmetry $A_{z z}$ in the quasi-elastic region [28].

\section{Summary}

Polarized solid targets, especially dynamically polarized ammonia targets, were utilized extensively during the seventeen years of CEBAF operations at Jefferson Lab. Four separate target systems were used to successfully complete more than a dozen experiments in each of Jefferson Lab's three experimental halls. Dynamically polarized ammonia $\left(\mathrm{NH}_{3}\right.$ and $\left.\mathrm{ND}_{3}\right)$ dominated the choice of target material, thanks to its superior performance in intense charged particle beams. Frozen spin targets of butanol and solid HD were used with secondary beams of tagged photons to exploit the higher acceptance afforded by the technique.

Polarized target usage during $12 \mathrm{GeV}$ era of Jefferson promises to be similarly rich. Although this era has not yet officially begun, more than a dozen experiments have been approved to use these targets, and the list is expected to grow. Once more, dynamically polarized ammonia promises to be the material of choice, although a substantial research program utilizing polarized solid HD has been conditionally approved in Hall D.

\section{Acknowledgements}

The author expresses his gratitude to all the scientists, engineers, and technicians of the Jefferson Lab Target Group, and to the past and present members of the University of Virginia Polarized Target Group. Particular thanks goes to Dr. Mike Seely, who led the JLab Target Group 1996-2008, and to Prof. Don Crabb of the University of Virginia. This material is based upon work supported by the U.S. Department of Energy, Office of Science, Office of Nuclear Physics under contract DE-AC05-06OR23177.

\section{References}

[1] T.D. Averett et al., Nucl. Instr. and Meth. A 427 (1999) 440.

[2] H. Zhu et al., Phys. Rev. Lett. 87 (2001) 081891.

[3] F.R. Wesselmann et al., Phys. Rev. Lett. 98, (2007) 132003.

[4] Jefferson Lab experiment proposal E07-003, https://hallcweb.jlab.org/experiments/sane/sane/docs/sane_update_pac31e.pdf

[5] Jefferson Lab experiment proposal E08-027, (http://hallaweb.jlab.org/experiment/g2p/docs/PAC33/dlt.pdf).

[6] Jefferson Lab experiment proposal E08-007, http://hallaweb.jlab.org/experiment/E08-007/home2_files/LowQ2Prop.pdf

[7] J. Pierce et al., Nucl. Instr. and Meth. A 738 (2014) 54.

[8] B.A. Mecking et al., Nucl. Instr. and Meth. A 503 (2003) 513.

[9] C.D. Keith et al., Nucl. Instr. and Meth. A 501 (2003) 327. 
[10] R. Fatemi et al., Phys. Rev. Lett. 91 (2003) 222002.

[11] Y. Prok et al., Phys. Lett. B 672 (2009) 12.

[12] S. Chen et al., Phys. Rev. Lett. 97 (2006) 072002.

[13] S.Pisano et al. Phys. Rev. D91 (2015) 052014.

[14] E. Seder et al. Phys. Rev. Lett. 114 (2015) 032001.

[15] C.D. Keith et al., Nucl. Instr. and Meth. A 684 (2010) 27.

[16] E. Pasyuk, Chinese Physics C 33 (2009) 1205.

[17] H. Dutz et al., Nucl. Instr. and Meth. A 356 (1995) 111.

[18] A. Honig, Phys. Rev. Lett. 19 (1967) 1009.

[19] X. Wei et al., Nucl. Instr. and Meth. A 526 (2004) 157.

[20] C. D. Bass, et al., Nucl. Instr. Meth. A 737 (2014) 107.

[21] M.M. Lowry et al., in Proceedings of PSTP 2013, PoS (PSTP 2013) 015.

[22] Jefferson Lab experiment proposal E12-11-108, https://www.jlab.org/exp_prog/proposals/11/PR12-11-108.pdf

[23] CLAS12: Longitudinally polarized target, https://www.jlab.org/Hall-B/clas12-web/specs/target.pdf

[24] Jefferson Lab experiment proposal E12-14-001, https://www.jlab.org/exp_prog/proposals/14/PR12-14-001.pdf

[25] CLAS12: Transversely polarized HDice, https://www.jlab.org/Hall-B/clas12-web/specs/hdice.pdf

[26] Jefferson Lab experiment proposal E12-14-006, https://www.jlab.org/exp_prog/proposals/14/PR12-14-006.pdf

[27] Jefferson Lab experiment proposal E12-13-011, https://www.jlab.org/exp_prog/proposals/13/PR12-13-011.pdf

[28] Jefferson Lab experiment proposal E12-15-005, https://www.jlab.org/exp_prog/proposals/15/PR12-15-005.pdf 